\section{Cahiers de Narratologie}

Analyse et théorie narratives

$10.1 \mid 2001$

La voix narrative

\title{
Voix/voie narrative : la voix métaphorée
}

\section{Mieke Bal}

\section{OpenEdition}

\section{Journals}

Electronic version

URL: http://journals.openedition.org/narratologie/6909

DOI: 10.4000/narratologie.6909

ISSN: 1765-307X

\section{Publisher}

LIRCES

\section{Printed version}

Date of publication: 1 January 2001

Number of pages: 9-36

ISBN: 2914561032

ISSN: 0993-8516

\section{Electronic reference}

Mieke Bal, "Voix/voie narrative : la voix métaphorée", Cahiers de Narratologie [Online], 10.1 | 2001, Online since 15 October 2014, connection on 23 February 2021. URL: http://journals.openedition.org/ narratologie/6909 ; DOI: https://doi.org/10.4000/narratologie.6909 


\title{
VOIX/VOIE NARRATIVE : LA VOIX MÉTAPHORÉE
}

\author{
Mieke BAL \\ Université d'Amsterdam
}

Qu'est-ce qu'une « voix » quand il s'agit d'un objet sans voix justement, un objet muet, texte dont seule la lecture peut faire un objet esthétique ? Une voix est corporelle, trace de la personne qui parle, moulage, miroir, index du sujet. Métaphore, le concept de voix mérite un examen critique. Mot, presque «naturelle » pour rendre compte que le récit ne vient pas de nulle part et que quelqu'un en est responsable, le concept paraît indispensable.

Un enjeu majeur, dans les débats qui ont suivi ma proposition d'amender la narratologie genettienne, concerne le caractère métaphorique des concepts que j'avais, à la lignée de Genette, simplement essayé de rendre plus « applicables ». Aujourd'hui on voit mieux que cet enjeu comporte celui, plus important, de la portée éthique des concepts des concepts mêmes ${ }^{1}$. Celle-ci tient à son sens, ou plutôt, ses sens, impliqués par la nature inévitablement métaphorique des concepts. Cela m'a été clair, même si insuffisamment, dès le début, mais je n'ai pas insisté assez sur la raison d'être de ce type de préoccupation dans la narratologie. Je n'ai pas assez insisté, je dirais maintenant, sur la portée idéologique du domaine esthétique, ni de son questionnement esthético-formel. Pourtant, c'est autour de la notion de responsabilité sémiotique formulée

${ }^{1}$ L'intérêt pour l'éthique - qui d'ailleurs cache mal celui pour la politique qui lui avait précédé - est vivant aujourd'hui. Témoin, par exemple, le numéro spécial de la revue canadienne Études littéraires publiée par l'Université Laval, intitulé "Éthique et littérature ». Dans ses pages, Pierre V. Zima semble considérer éthique tout ce que, il y a quelques années, on appelait encore politique. 1999 "Idéologie, théorie et altérité : l'enjeu éthique de la critique littéraire. "17-30 in Études littéraires 31, 3. 
dans les questions de base, introduites, puis désavouées par Genette : «qui parle? » et « qui voit? » que j'ai fini par développer toute une théorie du texte narratif contre le grain de l'entreprise genettienne. La question « qui » n'est pas seulement une personnification, sans doute problématique, d'un aspect textuel, mais aussi un début d'enquête, une question whodunnit, qui a commis ce crime, qui indique que s'il y a paroles qui portent, qui font acte comme nous l'a appris la théorie des actes du langage, il y a responsabilité pour ces actes.

«Qui parle ? " Ce sont les conséquences pour les deux domaines de questionnement qui m'ont d'emblée intéressé dans l'entreprise narratologique - le niveau de formalisation, donc, d'intersubjectivité, et la portée politique - de cette question et des termes d'analyse qu'elle implique, que je propose de soumettre à un examen critique. La question qui importe, je voudrais proposer, c'est la suivante, formulée d'une façon très simple: Qu'est-ce que cela veut dire de supposer que la narration possède une voix, et que celle-ci porte une identité ? La question qui, donc, et la nature du verbe parler. Cette question en contient à son tour deux : Qu'est-ce que cela signifie? - pour éviter la métaphore superposée dans la formule qu'est-ce que cela veut dire? - et quelles sont les conséquences ? Et ces deux questions, j'ajouterais volontiers, doivent se poser sur le mode positif aussi bien que négatif : quels sens et quelles possibilités critiques est-ce qu'on supprime en usant d'un concept comme la voix narrative ? En d'autres mots, c'est la question du statut du «métaphorique » dans les concepts, en général et dans le cas particulier de la voix narrative.

Pour des raisons entièrement aléatoires-l'écriture manuscrite de Gérard Lavergne ! - mon assistante à Amsterdam avait mis la présente conférence sur la liste de mes engagements de l'année, sous le titre "la voie narrative ». Un jour je me suis demandé quelle différence cela ferait à la narratologie si on remplaçait « voix-x » par "voie-e », une métaphore du sujet humain, anthropomorphe y compris le corps, par une métaphore spatiale. Voici donc le thème de ma communication, que je présenterai sous forme d'un petit nombre de thèses. Afin de 
dénaturaliser les deux mots de l'évidence, j'écrirai voix-x et voie-e tant que j'analyse les concepts, et simplement « voix » dans les autres cas.

1. Si on la maintient, la métaphore conceptuelle de la Voix doit être conçue dans une perspective historique.

Le discours presque dévot, mais aussi souvent un peu réactionnaire, qui réclame l'historisation de tout, comme si cela constituait une valeur naturelle, a quand même rempli le rôle utile de nous remettre les pieds sur terre après la folie de la généralisation du moment structuraliste. Aujourd'hui il semble tellement évident que toute analyse comporte une réflexion historique, qu'il me semble presque futile de le rappeler. Mais on historise peu les concepts. Historiser, il s'entend, n'est pas tracer l'origine, ni reconstruire la génétique de l'objet. C'est, plutôt, comprendre l'objet en vue de "son » moment historique : le moment où il a pris son sens plein. Bien sûr, cette thèse de bon sens requiert qu'on examine l'émergence du terme dans la critique littéraire, ce que je n'ai pas eu le temps de faire ; je dois donc me limiter à des spéculations. Si je raffolais d'origines - ce qui n'est pas le cas - je spéculerais donc que la métaphore est originaire de la période, autour des années vingt à 30 , de certaines découvertes et avances technologiques. Disons plutôt que c'est un moment qui la replira de sens et de pertinence ; c'est le moment qui pose le problème de la voix-x. En particulier, le concept de la voix-x entraîne des implications qu'on peut utilement comprendre à partir de la transition, plus lourde en conséquence qu'il n'y paraît à première vue, entre le film muet et le film sonore, transition qui a eu lieu à l'époque dite " moderniste. »

C'est une question de technologie tout d'abord. Avant, l'idée de pourvoir les images de son était une idée utopique bien exotique. Le mouvement était déjà un miracle assez impressionnant, pour l'appréciation duquel des peintres comme Degas, et surtout des photographes comme Eadweard Muybridge et, en France, Etienne-Jules Marey, avaient préparé le public ${ }^{2}$. Pour tourner des expériences techniques

2 Voir Mary Ann DOANE 1996 «Temporality, Storage, Legibility : Freud, Marey, and the Cinema " Critical Inquiry 22 (winter) : 313-43. 
en spectacle, on mettait un piano dans la salle de projection. Le son était un supplément de luxe, décoratif. Mais un jour, la technique a permis ce passage que nous trouvons tellement naturel, du film muet au film sonore.

Le moment qui m'intéresse, dans tout cela, est celui où l'on a commencé à ajouter du son. Le procédé d'ajouter un dialogue à un film muet s'appelait "goat-glanding " ${ }^{3}$. On connaît le phénomène, ne fût-ce que dans sa version fictionnelle proposée une génération plus tard, déjà nostalgique du jeu sans paroles, dans le film Singin'in the Rain de Stanley Donen and Gene Kelly (1952) où Debbie Reynolds agit comme prothèse acoustique à l'actrice «muette »-dont la voix ne passe pas - et emporte la victoire définitive. Ce procédé ouvrit la possibilité d'un nouvel engagement du texte avec l'image, engagement qui a fait du cinéma ce troisième art qui a mis en question l'essentialisme qui cherche à séparer les médias de façon absolue. Désormais, les études séparées des images - dans l'histoire de l'art - et des mots - dans les études littéraires et la linguistique -encourent le risque de développer un essentialisme, une attitude défensive et une fermeture aux influences dites "extérieures », dites « irresponsables » parce que "sans expertise spécialisée ». En d'autres mots, avant leur fondation comme disciplines vraiment autonomes, dans l'après-guerre, nos disciplines étaient déjà désespérément datées, fermement enfermé dans une situation passée et se fermant fermement les yeux au contemporain.

Mais cette situation culturelle, où le cinéma était le modèle culturel que nous ne commençons qu'à lui reconnaître, tant dans sa rupture avec l'idée de média " purs » que dans l'accession du grand public au statut de consommateur et juge de l'art, a aussi entraîné une crise. Cette crise mène à ma deuxième thèse :

2. Le concept de "voix narrative" est un instrument mis au service de la répression de la crise de l'autorité de l'auteur.

3 Sur la question de cette transition et le goat glanding en particulier, voir Tim Armstrong 1998 Modernism, Technology and the Body: A Cultural Study. New York : Cambridge University Press. 
C'est la crise de l'autorité de l'auteur, péniblement survenue à l'intérieur du fait technologique. Car dans les films soumis au " goat glanding » la mise en scène théâtrale, visuelle, du spectacle précède et domine le dialogue qui devrait être adapté à l'image déjà existante. La voix, instrument subordonné au service de l'image, était, en outre, l'instrument sonore de quelqu'un d'autre, puisque les acteurs souvent n'étaient pas doués pour la diction. Barthes et Foucault, en d'autres mots, n'ont tiré que les conséquences philosophiques du changement technologique, et ce, fort tard, avec leurs idées de la «dispersion» (Foucault) et «mort» de l'auteur. Le moment de la crise - moment bref, passant comme un éclair mais non sans laisser des cicatrices - était survenu quarante ans plus tôt. Crise de cette concaténation des quatre "facteurs de la vérité " inhérente à l'idée même d'auteur comme " voix ", justement, pourvue d'autorité, pourvue du pouvoir de se dire comme sujet, intention, origine et cause de l'œuvre. Soit dit entre parenthèse : si j'ai insisté, dans mes travaux de narratologie, sur l'importance centrale et structurale de la subjectivité, c'était en raison, justement, de cette répression, et non pas en la répétant. J'ai voulu détacher la question du sujet de celle de l'intention, de l'origine et de la cause, pour la réorienter non pas vers la génétique de l'œuvre mais vers sa destination. Je n'étais pas seule à vouloir séparer subjectivité de causalité et intention, loin de là. Mais paradoxalement, l'opposition furieuse à cette insistance cachait mal un attachement à l'humanisme contre lequel, justement, le terme même de narrateur - d'un narrateur pourvu, bien sûr, d'une voix - avait été proposé. D'où ma troisième thèse :

3. Le concept de voix narrative risque d'être mis au service d'une récupération de l'autorité de l'auteur.

Restons encore un moment avec le cinéma fraîchement pourvu d'une voix ${ }^{4}$. Bientôt, le dialogue parlé, ajouté aprèscoup ou non, devenait partie intégrante de l'œuvre cinéma-

${ }^{4}$ Je dois mon intérêt pour la différence qualitative radicale entre le film muet et le film sonore aux travaux de Nanna Verhoeff sur le premier. Voir son ouvrage (en préparation), After the Beginning: Cinema and the American West Before 1915. Utrecht, Cinema Studies. 
tographique, et la voix devenait porteuse d'un réalisme, à son tour instrument rhétorique qui garantissait l'authenticité comme effet. Pour remédier à cette naturalisation de la voix, porteuse d'autorité car de réalisme, je proposerais une poétique «techno »-c'est-à-dire, basée sur le sens matériel dont le concept avec son sens plein provient. La métaphore de la voix dirigerait l'attention, par exemple, sur la production du chronotopos diégétique comme domaine de l'effet du réel. Loin de posséder une autorité qui «va sans dire », la voix narrative "ajouté » détourne l'attention du manque total d'autorité pour implémenter la fiction diégétique comme cadre qui tirera le récit dans un trou chronotopologique dont, en général et sauf expérimentations postmodernes, il ne sortira plus.

C'est une belle métaphore que de parler de la voix narrative sur le fond de ce développement, par exemple, de la voix qui dit "longtemps je me suis couché de bonne heure » et " aujourd'hui maman est morte" ou encore, "puis-je, monsieur, vous proposer mes services, sans risquer d'être importun ? ». On le sait : la première voix, productrice de la phrase la plus commentée de la littérature, pose la distance temporelle comme indéterminée, tout comme le « il était une fois " des contes de fées. Le passé du narrateur de $L a$ recherche, par conséquent, est enchâssé dès la première phrase dans une temporalité fictionnelle qui permet au personnage de rester enfant même quand il est vieux.

A la différence de la phrase de Proust, mais sans doute, avec l'usage du terme familier "maman » en référence à cellelà, la deuxième, ouverture de L'étranger pose la proximité temporelle, tout en précisant que l'unité de temps, lieu et action de la tragédie classique s'appliqueront au récit à venir. En effet, cette voix-x positionne le récit de Meursault en rapport rhétorique avec la tragédie classique, rafraîchie par l'existentialisme qui pose, justement, le problème de la responsabilité morale dans des termes nouveaux, adapté à l'aprèsguerre. Et si, aujourd'hui, nous voulons reconsidérer cette question à la lumière de la perspective postcoloniale qui n'admet plus l'aveuglement à l'anonymat et de l'usage exploitatif de " l'Arabe », c'est justement parce que la voix-x, dans ses actes de paroles si emphatiquement dépersonnalisés, 
avec « je lui ai dit que cela m’était égal » comme adage, est responsable, non pas seulement de l'effet-tragédie mais aussi de la complicité du lecteur qu'elle sollicite. D'où ma quatrième thèse :

4. Le concept de la voix-x doit être historisée, spécifiquement à travers le model-métaphore du cinéma sonore, afin de pouvoir mener à une compréhension de la rhétorique proprement narrative sans adoption de l'autorité.

Et propre, bien entendu, n'est pas exclusive. Le "propre » appartient à, sans être possession de, ce de quoi il est " propre. » La voix-x « ajoutée » de La chute est dans ce sens un complément indispensable à celle de L'étranger, car elle, loin de faire appel à l'intimité familiale pour cadre où le conte de fées et la tragédie puissent s'installer subrepticement, met sur la table la question de la complicité. La voix-x « à la deuxième personne » montre bien, si besoin était, à quel point la grammaire est ici mise au service de la rhétorique ${ }^{5}$. Le critique belge-américain Paul de Man - lui-même, d'ailleurs, personnage principal dans un débat virulent sur la responsabilité de la "voix-x » lors de la découverte de ses écrits journalistiques de la période nazi - appelle cette imbrication de la grammaire et de la rhétorique « la rhétorisation de la grammaire et la grammaticalisation de la rhétorique $»^{6}$. Pour de Man, l'enjeu était la tension irrésoluble entre le mode narratif, essentiellement assertif - la rhétorique du vrai-faux, qu'il analyse à travers le passage sur le ruban volé dans Les confessions de Rousseau. Pour moi, la question de l'éthique n'est pas autre, mais est, au contraire, d'importance cruciale pour une évaluation esthétique des textes ainsi que le l'esthétique en soi. Elle ne peut pas être posée comme élément isolé, mais résulte de l'examen préalable des facteurs

5 Paul de MAN 1979 "Semiology and Rhetoric." In Allegories of Reading: Figural Language in Rousseau, Nietzsche, Rilke, and Proust, 3-19. New Haven, CT, and London: Yale University Press.

6 Paul de MAN 1979 "Semiology and Reading (Proust)." In Allegories of Reading: Figural Language in Rousseau, Nietzsche, Rilke, and Proust, 57-78. New Haven, CT, and London: Yale University Press. 
impliqués dans la métaphore conceptuelle de la voix-x. D'où ma cinquième thèse :

5. Transgressant ses propres limites mais ce, inévitablement, la poétique " techno" de la voix-x ajoutée pose la question de l'identité mais non pas aux dépens d'une compréhension de la rhétorique narrative.

Si de Man s'excite à propos de la tension entre les protestations d'innocence du Rousseau de l'épisode du ruban volé, et sa culpabilité narrative, c'est que ces deux ne sauraient être séparés, même pas pour le bien d'une mise en lumière de cette tension. Car la question «qui parle » est déjà inhérente au récit. Inévitablement, donc, ces voix-x impliquent aussi des déterminantes d'identité. Là encore, la révélation littéralisée par le levé du rideau, de la voix ajouté de Singin'in the Rain peut servir de modèle. L'identité de celle «qui parle » se montre, dans une mise en scène mise en abyme de la question «qui parle? » La scène-dénouement du film montre que la question importe ; le concept de la voix-x en dépend. Car la lecture des phrases inaugurales déjà citées de la perspective de la voix ajoutée nécessite sa propre transgression, son propre levé du rideau prématuré. Ici, la mise en scène « explique » pourquoi, dans l'histoire du cinéma, le caractère artificiel, non-identique, " ajouté » justement, de la voix-x a été « oublié » si facilement, si rapidement, peut-être si désespérément.

La première, celle qui ouvre La recherche, s'identifie comme voix d'adulte épousant la focalisation d'enfant, la deuxième s'identifie en situation familiale, sans autres précisions. La voix dans l'ouverture de La Chute, par contre, enferme la situation narrative dans le hic-et-nunc du présent, posant la complicité imposée entre la première et la deuxième personne ${ }^{7}$. Camus, en 1956, c'est-à-dire, à la même époque du film-modèle, détourne l'extase populaire devant la technologie pour poser des questions basales sur la complicité qu'engage tout lecteur. Contre ce fond, où la poétique « techno » entraîne sa propre transgression, l'identité jamais

${ }^{7}$ Edward W. SAID 1985 Beginnings : Intention and Method. New York: Columbia University Press. 
révélée du narrateur du roman El hablador de Mario Vargas Llosa, imbriquée dans la question de la "nation ", du colonialisme et des habitants indigènes du Pérou, et du problème de l'identité qui résulte de l'hybridité sociale - fondée, justement, sur l'oubli - est quand même posée dans la phrase inaugurale : "Vine a Firenze para olvidarme por un tiempo del Perú y de los peruanos y he aquí que el malhadado país me salió al encuentro esta mañana de la manera más inesperada. » (Je suis venu à Florence pour oublier-en espagnol, plus pertinemment, "m'oublier de "- le Pérou et les péruviens pour quelque temps, et ce fut ici que le pais malencontreux m'est sauté dessus ce matin de la façon la plus inespérée possible. $)^{8}$

Comme effet de rhétorique, la voix-x prise comme techniquement "ajoutée " plutôt que comme "source", origine, cause, intention du récit, a besoin de prendre le lecteur au piège, mais justement parce qu'elle n'est pas « naturelle »; son effet est construit, au dépens de la visibilité de sa construction que le levé prématuré du rideau dans Singin'in the Rain avait trahie. L'identité - «qui parle? » -se présente dès lors comme "ajoutée ", comme aspect indispensable mais toujours sujet au questionnement. Le cadre de la poétique « techno » sert à mettre en lumière ce fait socialement capital que l'identité est prothèse. D'où ma sixième thèse :

6. La voix-x est indispensable en tant qu'ajoutée pour le positionnement de l'identité comme prothèse: supplément, artifice, mais sans lequel le récit ne saurait " marcher».

Dans les cas cités, la perspective technologique modelée sur le " goat-glanding " permet de se rendre compte à quel point cette voix-x est une espèce de prothèse, une machine ajoutée mais indispensable pour le bon fonctionnement du récit. A propos de cette addition transgressive de la question de l'identité, je me plais à penser que c'est en la considérant comme effet, secondaire mais inévitable et en effet indispen-

${ }^{8}$ Sur ce roman en relation avec la question «qui parle? " comme enjeu de la confusion des nationalismes, voir Jonathan Culler, "Anderson and the Novel " in Diacritics (en préparation). 
sable, de la rhétorique de l'effet du réel produite par l'effet techno, qu'elle se pose. Dans ce sens, ou plutôt, à cette condition, la métaphore de la voix-x est indispensable.

Au cinéma d'antan, cette technologie avait sa matérialité propre : son, musique, appareil. Mais une compréhension cognitive inédite sous-tendait cette matérialité même, dont elle avait rendu la conception possible. La compréhension est fermement ancrée dans les sciences de l'époque. Elle concernait, non pas la surestimation, mais au contraire, le déficit fondamental du corps, nécessitant les prothèses de toutes sortes. Dont la voix. On n'a qu'à penser à Edison, personnage principal dans l'invention du cinéma, et producteur actif de films muets, inventeur, aussi, du stockage du son et de son usage à retardement, mais non pas de la voix ajoutée. Comme par hasard, Edison, introduit comme «l'homme qui a fait prisonnier l'écho », est aussi le personnage principal de L'Eve future de Villiers de l'Isle-Adam, roman qui prédate l'invention du film sonore mais coïncide avec les débuts du film muet. Roman dont la première phrase - «A vingt-cinq lieues de New York, au centre d'un réseau de fils électriques, apparaît une habitation qu'entourent de profonds jardins solitaires »- pose le discours cinématographique muet comme cadre. Elle le fait d'une façon que celle du Père Goriot ne pouvait faire, malgré la ressemblance superficielle entre les deux débuts.

Et pourtant, le roman de Balzac était un prédécesseur avec lequel l'ouverture de Villiers fait entendre une résonance par la description en zoom qui, chez Balzac pré-cinéma nécessitait encore un focalisateur-promeneur. Chez Villiers et son Edison il n'est plus besoin d'une telle incarnation de l'œil, et plutôt que de se révéler à l'œil du promeneur, la maison, ici, apparait au levé du rideau, cette fois moins prématuré que trop total, mettant à nu les fils qui font marcher la machine - et le roman.

La focalisation, porteuse de la vue, utilement dégage la voix-x du fardeau du corps. La voix-x comme prothèse, machine ajoutée, en effet peut être utilement déployée comme concept historisant et analytique à la fois, dans une narratologie doublement "appliquée » : au domaine littéraire, mais aussi appliquée sur elle-même, prenant au sérieux ses termes 
comme langage virtuellement créateur de fictions. Et ceci m'amène à une autre série de réflexions, d'un autre ordre. Les remarques suivantes ne concernent pas l'historisation indispensable du concept de la voix-x mais le fondement de ses implications théoriques dans la littérature même ; inversion donc du rapport théorie-analyse qui pour moi est très important du point de vue méthodologique.

7. Pour comprendre le concept de la voix-x et ses implications, la littérature est une ressource théorique indispensable : elle est un objet théorique.

La voix-x-prothèse est détachable du corps, et c'est en tant que telle qu'elle est soumise à l'examen de l'étonnement mise-en-scène dans ces mises-en-abyme dont Proust fut le maître. Nouveau au phénomène du téléphone aujourd'hui presque relégué au passé, remplacé par l'internet et le courrier électronique, le narrateur exploite l'étonnement comme procédé de production littéraire. Ainsi il performe (sens anglais) l'historisation dont il est question dans mon analyse théorique.

L'étonnement devant la voix de sa grand-mère détaché de son corps, de son visage, redéfinit le dernier cognitivement, justement parce que la routine perceptuelle a été éliminée par la technologie. Il est aussi étonné d'entendre une voix sans corps venant de loin que les spectateurs des premiers films, encore muets, ont été devant les images de visages agrandis et coupés des corps, ou des corps sans pieds. Il s'agit de l'aliénation qui résulte de la fragmentation du corps en fonctions séparées et isolées, fonctions, justement, dont la faillite peut être "supplémentée " par une prothèse. C'est en particulier cette dernière possibilité - du supplément technologique - qui impressionne et chagrine le narrateur, car c'est là que réside l'effondrement de l'effet du réel, dont l'artifice, par conséquent, devient apparent.

Avec une conscience douloureuse des intempéries de la perception, Proust écrit :

... tout d'un coup j'entendis cette voix que je croyais à tort connaître si bien, car jusque-là, chaque fois que ma grand'mère avait causé avec moi, ce qu'elle me disait, je l'avais toujours suivi sur la partition ouverte de son 
visage où les yeux tenaient beaucoup de place ; mais sa voix elle-même, je l'écoutais aujourd'hui pour la première fois. (II 134-35 ; italiques ajoutées)

La voix de la grand'mère est comme une photo d'elle telle que Proust définit la photographie ailleurs. Passage miseen-abyme symétrique à celui-ci, où il met en scène le focalisateur démuni de sa routine perceptuelle :

De moi - par ce privilège qui ne dure pas et où nous avons, pendant le court instant du retour, la faculté d'assister brusquement à notre propre absence - il n'y avait là que le témoin, l'observateur, en chapeau et manteau de voyage, l'étranger qui n'est pas de la maison, le photographe qui vient prendre un cliché des lieux qu'on ne reverra plus. Ce qui, mécaniquement, se fit à ce moment dans mes yeux quand j'aperçus ma grand-mère, ce fut bien une photographie.

(II 140/II 440)

Le passage célèbre se termine sur le résultat de cette focalisation défectueuse, résultat extrêmement perturbant dans sa négativité. Il se développe vers un langage de plus en plus hostile, voire violent, pour aboutir, au terme de cette gradation inquiétante, à la description de cette photo mentale qui accompagnera toujours le narrateur :

j'aperçus sur le canapé, sous la lampe, rouge, lourde et vulgaire, malade, rêvassant, promenant au-dessus d'un livre des yeux un peu fous, une vieille femme accablée que je ne connaissais pas. (II 141/II 440)

Comparé à cette photo inquiétante, la voix détachée du corps est porteuse d'une réalisation positive au-delà du chagrin de la distanciation aliénante : «.... ce que j'avais sous cette petite cloche approchée de mon oreille, c'était .... notre mutuelle tendresse. » (II 135)

L'aliénation est due au détachement, à la fragmentation du corps, d'une part, et à la distance à travers laquelle la partie du corps voyage, s'éloignant du tout, ou du reste. Cette distance, bien sur, n'est pas géographique mais ontologique, une distance entre la routine de la perception enchâssée dans l'affection, où l'affection constitue le cadre dans le sens derridien de parergon. Ce cadre est diffus, supplément indispensable sans lequel nous ne saurions vivre, précisément 
parce que le corps, dont intégrité et la totalité sont illusoires, a besoin d'extensions de cette sorte. Nous sommes tous des infirmes cognitifs, ayant besoin d'instruments - par exemple, des lunettes - pour pouvoir sortir de nous-mêmes, vers les autres.

Mais la métaphore proustienne, comme d'habitude, n'est pas que ce qu'on aurait tendance à penser, et c'est pourquoi je la prends comme apportant la théorisation - comme objet théorique. Ce qu'elle implique, dans son extraordinaire renversement de la perspective, est que ce n'est pas le téléphone qui est la prothèse technologique, le supplément fabriqué par l'invention nouvelle, mais le visage. Le visage, porteur, selon toute idéologie individualiste, des marques indélébiles de l'identité ; source et sauve-garde de l'unicité de chacun ; solide fondement de la personne dont la voix n'est que la trace littéralement distante. Ce visage n'est que la partition, le support matériel, l'outil qui permet de lire la voix. Qu'est-ce qui est donc prothèse, trace, moule de l'autre, la voix ou le visage?

Prenons, comme il se doit, les deux passages comme mises-en-abyme, ou mises-en-scène, de la conceptualisation, en narratologie, des deux catégories personnifiées de la voix et du focalisateur. Pour s'en tenir au thème de ce volume, nous avons ici une première indication de ce que la voix-x prend son sens « techno », c'est-à-dire, matériel et technique, dans la mesure où elle se définit comme voie-e : traversant la distance, la voix-x est capable de fonctionner comme une prothèse efficace permettant de produire une fiction qui révèle la «vérité » cognitive, tout cet appareil, ce dispositif, que les «vierges Vigilantes» ... « les servantes toujours irritées du Mystère, les ombrageuses prêtresses de l'Invisible, les Demoiselles du téléphone ! " (133) manipulent, telles un narrateur qui tire les ficelles derrière la cloison mince du théâtre de marionnette - ou une fée dans un conte de fées.

En effet, cette voix-x qui profère les mots « longtemps je me suis couché de bonne heure " met en scène un récit modelé sur le conte de fées où tout est possible, parce que - et c'est là ce que nous permet de voir la poétique " techno » de la voix artificiellement « ajoutée » au film/photo muet - la voie-e, la distance à traverser, est couverte, automatisée pour 
ainsi dire. Car la voix-x a été privée de son corps. C'est là le sens du visage-partition de la grand'mère. La voix-x voyage tout seul. C'est là la source de l'étonnement du narrateur proustien.

Restituons donc, juste pour voir, ce corps du narrateur porteur ou origine de la voix. Son sexe, son âge, sa position sociale : tout cela n'est pas naturellement excisé de notre concept. C'est un effet de l'analyse, donc, du concept déployé dans son service, que de considérer ces aspects nonpertinents. La voix-x narrative, serait-elle donc voix sans corps, monstre pourvu d'une voie-e à la place d'un corps ? Et pourtant ...

8. Le corps auquel la voix-x appartient n'est ni naturellement pertinent, ni évidemment non-pertinent; il est sujet à l'examen critique auquel est sujet le concept de la voix-x.

Certaines œuvres se comprendraient peut-être mieux si l'on prenait le concept métaphorique à la lettre. «Elle marche, écrit Peter Morgan », première phrase, encore, cette fois du Viceconsul de Duras, auteur qui épouse l'étonnement de la leçon de Proust pour établir une distance visible dans tout son tragique, entre l'Anglais narrateur de fiction et le personnage oriental, féminin, affamé qui sort de sa plume, mais qui, transgressant la convention des niveaux narratifs rassuramment distingués, entre dans le jardin bien maintenu de l'ambassade.

$\mathrm{Ou}$ alors, celle-ci, qui ouvre Le ravissement de Lol V. Stein : «Lol V. Stein est née ici, à S. Tahla, et elle y a vécu une grande partie de sa jeunesse. " Le narrateur de ce romanlà se trouve bien avoir un corps quand, plus tard, beaucoup plus tard, il se retrouve avec Lol dans une chambre d'hôtel. La question du corps dans lequel loge la voix narrative a été mise en scène - cette fois, littéralement pour ainsi dire - par l'artiste irlandais James Coleman dans une installation intitulée Photograph («Photographie ») de 1998-1999.

L'installation consiste en une série de diapositives, projetées dans un spectacle durant 19 minutes, et accompagnées d'une voix lisant, sur un ton théâtral et très lent, un texte de nature romantique. La voix, ici, une voix-off, est jeune, féminine, dramatique sinon sentimentale, contrastant 
maximalement avec celle qu'aurait un narrateur " autobiographique » identifiée à l'artiste qui, lui, est homme, approchant la soixantaine, et très terre-à-terre. Cette voix-x off est en effet off, éloignée dans le temps, dans la mesure ou nous ne sommes plus à l'époque romantique, et éloignée dans l'espace, elle est sans coïncider avec l'image qui serait son objet, ni avec l'auteur dont l'autorité la «couvrirait », dans une conception autoritariste de la voix-x.

Le texte lu, à son tour, contraste fortement avec les images d'adolescentes préparant, dans une école de banlieue de classe ouvrière, un spectacle de danse. Les scènes photographiées n'ont rien de romantique, mais on ne peut pas non plus ignorer l'effet émanant de la juxtaposition de la voix et des images, qui se supplémentent mutuellement. La voix est " coloriée " par les couleurs brillantes des photos, par exemple; les images acquièrent une dignité historique et une lourdeur émotive conférée par la voix. Mais malgré les rapprochements qui s'imposent à force de rester devant, ou dans, ce spectacle "son et lumière ", la voix reste, inexorablement, off.

Les contrastes ont pour effet d'incarner, ou de mettre en scène, une position théorique qui pose le discours comme " sans voix ", diffus, hétérogène, comme des fragments flottant dans la culture d'aujourd'hui comme autant de morceaux détachés d'un passé culturel dont la mémoire, diffuse, est encore avec nous. L'auteur - l'artiste Coleman qui s'est appuyé tellement sur le discours dont il cite des bouts de phrases qu'il se considère comme scripteur, refuse de donner accès au texte écrit, et insiste sur l'anonymat du discours dans son œuvre. Et il a raison. Le discours est romantique, mais les fragments de texte sont inidentifiables. La rime et le rythme ne sonnent pas exactement comme Yeats ou Coleridge, même si on pense à ces poètes, inévitablement. Le charge émotif est là, mais les émotions ne sont pas représentées.

En vue de cette fiction qui proclame la désincarnation de la narration dans le geste même qui marque le corps du sujet narrateur - le manque de voix- $x$ dans la mesure de la pratique citationnelle comme modèle - on se voit amené à vouloir prendre une position dans le débat implicite qui sous- 
tend une telle attitude. D'un côté, donc, le concept de la voix$\mathrm{x}$ narrative est construit sur le présupposé de la voie-e ; telle, du moins, est la leçon de l'étonnement de Proust devant le téléphone. La voix-x perd son corps mais, en gagnant la distance elle gagne aussi un pouvoir sur la démarche, le parcours, la mise en place du récit - et voici que j'utilise trois notions qui sont à leur tour, entièrement spatiales. D'autre part, le même concept désavoue justement le lieu d'origine de la voix-x : ce visage que Proust propose comme prothèse disparaît, mais dans la tentative même de l'incarner, de lui rendre son corps, en marquant le sexe, l'âge, et la position sociale ou même nationale - ici, et en résonance avec la phrase de Vargas Lllosa, la voix-x de l'œuvre de Coleman a un très léger accent irlandais.

S'il est permis de simplifier l'aspect historique de cette analyse, on peut dire que le moderniste Proust tente désespérément de sauvegarder la "personnalité », le caractère personnel de la voix- $x$, la tendresse mutuelle entre lui et sa grand'mère, d'autant plus « lisible » dans la voix-x que la distance - la voie-e - a coupé le lien avec la «partition » qu'est le visage. L'artiste postmoderne, par contre, restitue le corps et refuse l'origine, l'idée de la voix-x comme point unique où le récit prend son essor. Paradoxalement, dès lors, la personnification qu'on trouve dans le concept - son caractère décrié par les puristes de la science - est ici mise en scène en réponse à la dépersonnalisation de la technologie qui sépare voix du corps. Pour anticiper mes conclusions : je crois qu'il est important d'écouter l'enseignement de ces œuvres esthétiques, en même temps que de continuer à théoriser, mettant l'art et la théorie en conjonction l'un avec l'autre, en dialogue sinon en dialectique, mais sans solution transcendante. La raison est la suivante.

9. La séparation non-raisonnée de la voix du corps, dans la résistance au caractère métaphorique du concept, risque de séparer sens et signification, du désir et de la violence.

Et cela, ce serait une censure de l'analyse, censure qui combat la perspective proprement critique qui, étymologiquement, en est pour ainsi dire le synonyme. Ana-lusein comme kritein signifient débrouiller, si je puis me permettre de continuer 
une étymologie un peu tongue-in-cheek. Le professeur mythique de l'impossibilité de cette séparation est la figure de Lucrèce dans les textes de Tite-Live et d'Ovide, dont il ne faut pas oublier l'emprisonnement dans la voix-x de mythographes.

A quelques lignes de distance le personnage de TiteLive, mis en scène, est cité comme se prononçant deux fois sur la question de cette séparation, et les deux énoncés sont en contradiction logique l'un avec l'autre, dans un langage que je traduis en résonance avec ce christianisme qui en a profité :

- Seul mon corps a été violé; mon esprit est sans culpabilité

- Même si je m'acquitte du péché, je ne m'absous pas du châtiment ${ }^{9}$

Entre ces deux énoncés se situe l'abîme qui clive le sujet en deux. Inutile de dire que dans le cas de Lucrèce, c'est le sujet social, subordonné aux lois du patriarcat à son plus virulent, qui l'emporte. Pour la question de la voix-x et son rapport avec le corps, il importe de mettre l'accent sur la voix$\mathrm{x}$ narrative qui « cite » la victime.

Les paroles de la femme sont mises en scène à l'intérieur d'un double abus du corps féminin, d'abord comme occasion de viol, conçu comme vol du mari, donc, comme rivalité entre hommes, ensuite comme occasion de révolution politique, usage du corps violé et suicidé comme appel à la révolte. C'est dire que le personnage qui incarne - c'est le cas de le dire! " la problématique de cette séparation, est aussi l'occasion de sa propre défaite comme parleuse. Pour donner toute sa force littérale à un concept théorique, c'est une miseen-abyme-en-abyme.

Rassurez-vous ; ce n'est qu'un exemple. Le rapport entre voix-x et corps est à la fois plus banale, plus générale, et plus indispensable. Il comporte la régularisation par le ritme, aspect narratif dont c'est encore Genette qui a évoqué l'appartenance à la narratologie, encore, sans lui donner sa place dans une systématique du texte narratif comme objet

9 Je me suis appuyée sur l'édition de B.O. Foster 1959 Livy, with an English translation by B.O. Foster. London: W. Heinemann Ltd.

Heinemann Ltd., p. 203, traduction adaptée. 
sémiotique. Systématique qui « j'espère que c'est clair maintenant » je considère comme à la fois indispensable pour éviter les répressions et les prises au littéral » des facteurs du concept, et, en même temps, hautement douteux et donc, constamment sujette au questionnement critique. Ce que j'aimerais quand même retenir, de la voix-x, c'est le rapport - rythmique - entre la voix et le mouvement du corps.

Il est grand temps que je m'arrête là, dans cette révision que je voudrais constructive, du concept de la voix-x, car il n'y a pas que des avantages à notre concept. Ma dixième thèse, par contre, égrène les aspects dont on ferait bien de se méfier, même si, encore, on ne s'en débarrasse pas si facilement que ça. Mon plaidoyer? Qu'on continue d'utiliser le concept de la voix-x ; qu'on lui conserve même les aspects métaphoriques qui sont si gênants; mais qu'on se rende compte de ces aspects, de sorte que leur invocation pourra se faire explicitement, à des buts précis, et avec des restrictions claires.

10. La voix-x risque de conserver, dans la théorisation de la narration, les éléments métaphoriques qui ont motivé l'invention du concept.

Essayons donc de mettre "sous rature » les sens suivants. La notion du sujet comme propriétaire ou site de la voix narrative risque de privilégier la voix-x au-dessus d'autres éléments à force sémiotique, tels que, pour ne donner que l'exemple le plus évident, l'image. S'il y a une leçon utile pour les études littéraires et en particulier pour la narratologie appliquée, qu'on peut tirer de l'intérêt actuel pour l'image, ce n'est certes pas qu'il faille abandonner le littéraire pour nous tourner tous en historiens de l'art manquant de formation. Non, c'est de prendre en considération la possibilité que le texte n'est pas réductible à l'ensemble des mots qui le constituent. L'image est un élément de tout récit, avec lequel la voix-x entretient une relation mais qui n'est pas systématiquement de maîtrise. Le heurt épistémique, poétique et libidinal qui caractérise le récit proustien en est encore un exemple, auquel toute analyse de la voix narrative dans $L a$ recherche aussi bien que toute analyse de la peinture dans le 
roman, continuent de rester aveugles ${ }^{10}$. L'étude de l'image « vraie », l'image objet (d'art) visuel, utile en soi bien sûr, peut aussi éclairer utilement ce rapport ambigu et troublé entre image et langage que Photograph de Coleman met en scène avec tant de précision.

Ce privilège accordé presque sans réflexion à la voix-Xsujet entraînerait une mimésis extrémiste, un simulacre des rapports sociaux que la littérature a quand même pour mission de questionner"1. La question "qui parle?" nécessite la question complémentaire, «qui ne parle pas ? » quel personnage, dans quelle position sociale, n'a pas accès à la parole ? Comme le fait remarquer la critique Gayatri Chakravorti Spivak, dans une analyse percutante du cas du "vendredi » de Daniel Defoe, revisité par Coetzee dans son roman Foe, la langue de Vendredi a été coupée ${ }^{12}$. Dans la version postmoderne de Coetzee cette langage a été coupée littéralement, donc, physiquement. Torture qui, en soi, peut servir d'objet théorique, accentuant l'importance d'une perspective presque naïvement littéralisante dans une réécriture qui se veut désabusée du réalisme. Paradoxe important, il me semble, pour opposer la tendance néfaste et réactionnaire de disqualifier le postmodernisme comme exclusivement ludique, comme si le jeu était dangereux et la "réalité » sûre ! Je suis d'autant plus heureuse d'invoquer le livre de Spivak que

10 Pour une analyse détaillée, ainsi qu'une bibliographie, voir mon étude Images littéraires, ou comment lire visuellement Proust. Toulouse : Presses Universitaires de Toulouse le Mirail 1997.

11 Ceci constituait l'enjeu de ma dispute avec Genette autour de l'exemple de Verne qu'avait allégué le dernier dans Figures III, puis, après ma critique dans Femmes imaginaires. L'ancien Testament au risque d'une narratologie critique (1986 Utrecht: Hes Publishers/ Montreal : HMH Hurturbise/ Paris : Nizet) réitéré dans G. Genette 1983 Nouveau discours du récit. Paris : Éditions du Seuil. Disons - pour la dernière fois ! - que la subjectivité narrative - ce jeu complexe entre les différentes positions organisées autour de la narration et la focalisation - ne coïncide pas avec les relations sociales que le roman en question est censé représenter. La pertinence du récit littéraire réside justement dans ce refus d'obéissance à la pression du réalisme «trompe-l'œil » qu'est le simulacre.

12 Gayatri Chakravorty SPIVAK 1999 A Critique of Postcolonial Reason. Cambridge, MA : Harvard University Press (186-87). 
cette critique est une des rares personnes à savoir - et vouloir ! - intégrer à la perfection une perspective politique, celle de la critique postcoloniale justement, avec une perspective déconstructionniste extrêmement efficace qui lui interdit de tomber dans les pièges du concept de la voix-x inventoriés ici. C'est dire que, dans ses travaux mieux qu'ailleurs, l'inutilité, voire la nocivité de ce clivage si habituelle est minée à chaque page.

Ensuite, le sens d'autorité, déjà évoqué, autorité qui est à la fois oblitérée et protégée, détournée par la critique qui pourtant s'en autorise, pas la notion de l'intention. On sait que le concept de narrateur a été inventé pour éviter l'appel automatique à l'auteur pour aurotiser une interprétation. Néanmoins, le colloque de Nice a connu de nombreux moments où l'auteur se trouva invoqué à tout propos, comme si sa présence-fantôme pouvait trancher quelque question interprétative ou théorique que ce soit. Deux problématiques se dessinent ici. L'une concerne l'attribution de l'intention comme arme au service de la subordination du lecteur. Ce dernier, que l'enseignement a appris à intérioriser l'interdit d'exercer sa fonction de deuxième personne, s'incline trop facilement devant l'intention dont se revêt le texte conçu comme produit de la voix-x insuffisamment mise en doute.

La deuxième problématique concerne l'hypostase du critique qui, mystérieusement mis en état de parler " pour » l'intention, cache sa propre subjectivité, sa propre voix-x, derrière la puissance incontournable de la voix-x narrative qui, elle, dit la vérité sur elle-même. La question si fascinante du mensonge et de la mauvaise foi, au centre de la critique existentialiste aussi bien que déconstructiviste - témoigne l'insistance de Paul de Man sur l'épisode du ruban volé - n'a pas de statut narratologique théorisé tant que la voix-x retient les cordons de la bourse. Mais, au-delà de la thématique de la sincérité, l'authenticité, et la compétence narratoriales, le problème de l'unité et de la mémoire culturelle tel que l'œuvre Photographie de Coleman l'avait mise en scène ne permet pas l'unification de la voix-sujet sous l'adage de la voix-x. D'une part, cette œuvre démontre que la voix-x qu'on attribue au texte ne saurait être objectivée. A aucun moment le lecteur ne peut être certain que le texte qu'il entend est le 
texte dit. D'autre part, c'est justement dans la mesure où on est ainsi privé de l'autorité de la voix-x du scripteur qu'on se trouve confronté à la nécessité, donc, aussi, à la liberté accordée au lecteur de dire son mot.

Responsabilité, en d'autres termes - une responsabilité à laquelle je continue de tenir, avec Spivak, et malgré le travail utile du déconstructivisme - n'égale pas autorité ; subjectivité n'est pas agence, et, dans le sens contraire, l'agence - dans le sens anglais de agency - ne saurait profiter de la problématisation de l'unité du sujet pour se dispenser de la responsabilité.

La voix-x ne saurait pas non plus se proclamer de l'origine. La question "d'où viennent les mots? » ne trouve pas sa réponse dans le concept de la voix-x, même si celui-là insiste utilement sur la question. Il s'agit d'une perspective de générativité, qu'il importe de garder dans ses justes proportions. L'insistance sur l'illocution, et la subordination de la perlocution concomitante que cette perspective encourage, mérite d'être contrariée par l'appel à la grammaire que résiste - utilement aussi - Paul de Man. Appel à son tour problématique dans la mesure où la notion de voix grammaticale comme voix-x est tout aussi ambivalente, mais en même temps indispensable, car la voix grammaticale-active ou passive, ou, n'oublions pas, réfléchie et réciproque dans une approximation de la voix moyenne grecque ! - se supplémente de la catégorie également métaphorique de la "personne». La question "qui parle? », dans ce sens entraîne comme supplément la question « à qui parle-t-il ? » et qui, donc, est en position de s'approprier la voix-x et de parler en retour, de répondre ${ }^{13}$.

13 Pour l'importance systématique de la «deuxième personne ", importée dans la narratologie à la suite de la linguistique d'Emile Benveniste, voir mon ouvrage Double Exposures: the Subject of Cultural Analysis. New York: Routledge 1996, en particulier le chapitre 5. Je me réfère à l'ouvrage philosophique important de Lorraine Code What Can She Know? Feminist Epistemology and the Construction of Knowledge. Ithaca/London: Cornell University Press 1991. Sur l'importance de la voix moyenne grecque déjà invoquée par Barthes dans Le bruissement de la langue, voir Hayden White 1992 "Historical Emplotment and the Problem of Truth. " In Probing the 
La grammaire, toujours dans la perspective génétique mais déjà plus proche de la voie-e, est une structure formelle qui suit un parcours. Dans le chronotopos théorique où je me suis située d'emblée, la voix-x projette le récit. D'où les phrases d'ouverture que j'ai invoquées comme exemples. D'où, aussi, l'œuvre de James Coleman, corps étranger dans notre discussion, mais de ce fait même occupant une position de choix comme "voix » d'artiste-théoriqueur, si le jeu de mots m'est permis. Car sous sa guise d'objet théorique, cette œuvre se sert de la technologie franchement datée de la projection de diapositives pour insister qu'elle projette les images, tout en projetant, au futur de la réception de l'œuvre - le " récit » illisible qui, se situant dans le temps, a de ce fait "mérité » son statut narratif qu'elle refuse sur le plan diégétique ${ }^{14}$.

Un troisième aspect, rhétorique celui-là, de la perspective génétique, concerne le rôle inévitable de la métonymie. Au risque de paraître redondante, permettez-moi d'évoquer encore une fois le nom de Genette comme fondateur d'une certaine attention à la présence de la métonymie dans toute métaphore. A la lumière de la projection - et le joint entre lumière et projection est encore une contribution théoricoesthétique de l'œuvre de Coleman - la métonymie met en place la syntaxe rhétorique qui fait partie intégrante de tout récit comme son principe de lisibilité modale. Là encore, Paul de Man a repris a son compte cette attentivité, faisant de la métonymie et de la narrativité des synonymes, à travers la grammaire. Pour lui, métonymie et grammaire sont des faits de

Limits of Representation: Nazism and the "Final Solution", ed. Saul Friedlander, 37-53. Cambridge : Harvard University Press.

$14 \mathrm{Si}$ j'appelle Photograph un corps étranger, c'est pour démontrer l'importance à la fois du corps et de l'étranger pour une revitalisation des concepts narratologiques. En même temps, l'expression se réfère à une autre œuvre d'art visuel, également à forte portée théorique, Corps étranger de Mona Hatoum, actuellement exposée dans le musée du Centre Georges Pompidou à Paris. Cette œuvre fait l'objet d'une analyse, pertinente pour ma présente contribution, dans mon livre Quoting Caravaggio: Contemporary Art, Preposterous History. Chicago : University of Chicago Press 1999 (137-44). 
l'ordre des mots ${ }^{15}$. Pour moi, la métonymie ainsi conçue ajoute à la déconstruction de la catégorie de la voix-x qui ne saurait maîtriser l'ordre des mots, ni celui de la lecture.

Ensemble, donc, ces aspects mis sous rature garantissent l'inscription du temps comme fondation de la narrativité. C'est important. Je ne suis donc pas prête à laisser le concept de la voix-x succomber sous le poids de toutes ces critiques. Courage, mon concept! Il faut continuer. Mais cette temporalité se situe dans l'intérieur du chronotopos qu'est avant tout toute œuvre de narrativité, ce qui amène ma thèse onze.

\section{Ce qu'implique le concept de voix-x tout en l'oblitérant, c'est sa contrepartie spatiale, ou la voie-e.}

En effet, la métaphore conceptuelle réprimée par l'usage trop simplement anthropomorphe de la notion de voix narrative, c'est la voie-e narrative ${ }^{16}$. Pour embrouiller nos pistes à fond,

15 Je me permets de citer ici un passage d'une communication personnelle de Cynthia Chase qui dit mieux que je ne saurais le rendre l'enjeu de la pensée de de Man: The narrative voice is a sort of projected mirror image of the reader's (the reading's) two-step analysis, which can be felt as having a narrative pattern : first we see the metaphor, then we realize that its condition of possibility is a metonymy. That is, I'm saying that the "narrative " element there in the figure of "narrative voice ", at least in the kind of case de Man gives an example, is just as much a metaphor as the "voice " element. The self-deconstructive character of the text is personified as a narrative voice that comes to possess a deconstructive insight. The narrator is «the metaphor of a grammatical syntagm whose meaning is the denial of metaphor..." (18). (That projection of a mirror image of the reading is what de Man writes that "autobiography" entails and makes conspicuous, in The Rhetoric of Romanticism, New York: Columbia University Press 1984, ch. "Autobiography and Defacement. ")

16 Je remercie donc Sherry Marx pour sa petite erreur de transcription, Gérard Lavergne pour son écriture illisible, et Cynthia Chase pour avoir pris la transcription de Sherry au sérieux dans le courrier électronique cité dans la note précédente et qui a orienté l'inspiration de ma conférence. Ces trois remerciements, comme il se doit, accouplent la plaisanterie au sérieux. En effet, sans Sherry je ne pourrais pas fonctionner; sans Gérard, je n'aurais jamais réfléchi aux implications de ma propre terminologie ; sans Cynthia, je n'aurais pas $\mathrm{pu}$ intégrer la perspective déconstructionniste à celle d'une narratologie « applicable». 
donc, permettez-moi de terminer sur une esquisse rapide des aspects que la présence inévitable de cet « autre » de la voix$\mathrm{x}$ importe au sein de la catégorie de base de la narratologie appliquée, qui est le thème de notre colloque.

Tout d'abord, et encore, d'une façon à la fois évidente et étonnante - au sens proustien - on tiendra compte de la spatialité. C'est dire que la voie-e établit le texte narratif comme réseau. Ce n'est rien de nouveau; le structuralisme n'est pas loin. Réseau, par contre, qui est plus proche de la toile d'araignée que du réseau des chemins de fer. Il emprisonne, met en danger le lecteur qui aura du mal à se débarrasser de ces fils qui collent, mais dont on peut aussi se débarrasser. C'est encore une leçon de la voix-x théâtrale, déclamant les paroles sans voix-X-origine, de l'œuvre de Coleman : une fois pris dans la toile des paroles diffuses de ses lectures, on les porte en soi, sans pouvoir les maîtriser ni oublier, sans pouvoir leur attribuer une origine ni se les approprier ${ }^{17}$.

Le sens spatial de la voie-e milite aussi contre l'atomisation du sens, telle que l'implique la sémantique structurale. $\mathrm{Au}$ contraire, la voie-e propose une construction sémantique dont les briques sont des sens accumulatifs. La métaphore spatiale dit bien qu'en tant que lecteur, on parcourt le texte, mais à chaque étape on continue avec des bagages. Selon cette métaphore, la linéarité de la lecture se complique d'une " croissance » progressive mais en même temps non-systématique de « couches » de sens. La métaphore architecturale, ici, est à prendre au sérieux. Ce qui résulte de ce procès est bien un bâtiment, solide dans la mesure où on ne peut l'éliminer de la culture; fantaisiste dans la mesure où sa construction ne

17 C'est la raison pour laquelle Coleman a raison de refuser l'accès au texte même en vue de cet article. Il refuse, en d'autres mots, toute tentative d'attribution des paroles. Ces paroles appartiennent à la mémoire culturelle, par définition diffuse. Ce que j'en fais, moi, est donc nécessairement et systémiquement différent de ce que lui, Coleman, a écrit comme la fiction qui fait partie de Photograph. J'en fus tourmentée, mais c'est encore une dimension de Photograph-objet théorique dont j'ai appris des choses "par la chaire " (et ce, littéralement, comme j'ai eu du mal à prendre des notes dans le noir, debout, pendant longtemps. Cette expérience physique aussi fait partie de l'œuvre). 
répond que partiellement au plan de l'architecte-auteur. Ce qui importe le plus, ici, c'est la mémoire textuelle, qui n'est pas exclusive au récit mais dont la modalité narrative propose le modèle, mis en abyme génériquement pour ainsi dire par le roman policier.

Le sens spatial ainsi expliqué porte à conséquence. Car de son fait, le lecteur détient l'autorité narrative qui régularise la construction du sens. Comme le disaient déjà les théoriciens de l'esthétique de la réception, le livre non-lu reste livre mort, l'actualisation dans la lecture en fait texte, littérature. Le sens spatial de voie-e ainsi répond - comme deuxième personne qui ne réprime pas sa tâche - aux sens importés par la voix-x. Chaque lecture ajoute une ligne à cette carte géographique des sens. La fluidité de la présence culturelle des lectures - diffuses, multiples, mais auxquelles " le texte » ne se réduira jamais - est mieux comprise selon la métaphore spatiale de la voie-e que selon celle, temporelle et vite rendue linéaire, de la voix-x. Le temps de la mémoire contient le temps narratif et compose sa linéarité. Dans ce sens, j'irais même aussi loin que de dire que la temporalité, malgré tout constitutive du récit, est quand même subordonnée à la spatialité conceptuelle.

Comme aspect de cette spatialisation de la voie-e, la notion de mise en plan, selon la métaphore de la carte de géographie, la notion de "mapping " peut être intéressante. Elle se réfère au travail structurant dont la réalisation revient $\mathrm{au}$ " lecteur-voyageur » mais dans sa guise de touriste qui, tout en suivant les indications - de la voix- $\mathrm{x}$ - cherche à éviter les pièges à touristes, préférant explorer des terrains encore inconnus ou au moins rarement visités. Le comparant de la carte géographique comporte un "survol de terrain " dont les détails se révèlent à un rythme autre que la séquence linéaire le suggère. La tension entre ces rythmes différents contribue encore à la libération du lecteur de la tutelle injustifiée et néfaste de la voix-x-autorité.

Finalement, le mouvement du «corps narratif » fait symétrie avec le corps déjà évoqué à propos de la voix-xcorporelle. On n'a qu'à penser à la description narrativisante dans Madame Bovary de Flaubert, que je n'ai cessé d'invoquer depuis mes tout premiers travaux en narratologie, et à 
laquelle je ne résisterai pas davantage aujourd'hui. Car dans le cadre de mes remarques ici, la rhétorisation de la grammaire prend une allure fort belle comme corps textuel mouvant, lorsque l'illusionnisme romantique d'Emma se transmet au lecteur médusé par une faute de grammaire, lorsque la voix-x, mettant en chantier la voie-e, introduit la fameuse description de Rouen avec l'oxymoron grammaticale «tout à coup, la ville apparaissait. » La figure - car c'en est une - de rhétorique proprement narrative, intégrant grammaire et visualisation, fonctionne par de multiples connotations ou directions : la série de descriptions de lieux dans lesquels Emma projette ses désirs, tels le château de Vaubyessard, Yonville, et Rouen au départ ; la tension entre désir/espoir et désillusion/désespoir; la symétrie, aussi, entre ce bout de phrase "incorrecte » et le début du roman supercorrecte car justement, objet d'étude pour tout enfant français («Nous étions à l'étude, quand le proviseur entra»). Heureuse suppression du temps verbal connoté « littéraire », le passé simple, jugé trop simple ici, ainsi que de sa contrepartie dite "parlée », le passé composé ! Ce qui sort de la voix-x et déborde ses limites, c'est précisément une description qui fait image tout en faisant double voix- $\mathrm{x}$ - la doublure de l'ironie mais sans cible bien claire - dans l'acte même qui en fait voiee. Car à la fin de la description dite soudaine, le fiacre aura parcouru l'entrée de la ville, traversant le passage de la carte postale-illusion à la sordide réalité de l'ennui ${ }^{18}$.

Cette voix-x qui se soustrait au contrôle narratorial, pour risquer de s'égarer sur les multiples sentiers dont consiste la voie-e, c'est celle, ou celles - avec l'ironie, on ne sait jamais mis en plan si superbement dans un autre domaine " étranger » encore, celui du cinéma, par Kaja Silverman, dans un livre dont les implications n'ont pas été suffisamment saisies: The Acoustic Mirror. C'est une des études plutôt rares qui examinent à fond ce qu'est la voix-x, ce qu'est son sexe - hautement pertinent dans le cas de la voix-x - ainsi que son «âge", puisque la voix des premiers parents,

18 Sur l'ironie, voir l'ouvrage fondamental de Philippe HAMON, L'ironie littéraire. Paris : Hachette 1996. Voir aussi, dans ce volume, la contribution de Peggy Karpouzou. 
notamment de la mère, ont une si grande influence sur la formation du sujet. Pour finir sur un dernier double paradoxe : c'est dans cette étude sur la voix-x dans son sens littéral qu'on trouvera les indications nécessaires pour complexifier le concept de voix narrative en incorporant, dans ses multiples sens métaphoriques - mais y a-t-il d'autres ? - les aspects, indispensables pour une compréhension du dynamique narratif, de la voie-e. En outre, c'est là qu'on trouve une théorisation, pertinente à plusieurs niveaux, du théorème métaphorique du miroir : reflet mais non pas réaliste ; réflexion sans symétrie ; trace ou moulage, inversé au-delà de la reconnaissance facile ; index de la " personne » mais qui mine la définition même de la personne. Image-mise en abyme au-delà duquel le texte se soustrait à la saisie, désir inavouable du critique ${ }^{19}$.

Faut-il terminer, alors, sur une conclusion ? Je suis tentée de résister à cette convention. Car ce que j'ai cherché à faire, dans ces quelques remarques, c'est d'ouvrir le champ d'investigation sur lequel se joueront les débats des jours du colloque. Conclure, par contre, est une façon de fermer. Autrement dit, je n'ai pas voulu prescrire une approche, limiter la démarche, ni même exprimer une préférence pour telle interprétation, positive ou négative, du concept de la voix narrative. J'ai essayé de mené de paire la critique et la justification du concept.

Bien évidemment, on peut chercher à développer des métaphores conceptuelles alternatives, telle celle du rhizome proposée naguère par Gilles Deleuze. Ce concept, pourtant, ne mérite peut-être pas entièrement l'idéalisation que la popularité de Deleuze lui a conférée. Car s'il accepte la nonlinéarité, il continue, avec sa tige souterraine, de présupposer une origine commune, il implique l'organicisme hérité du romantisme, si efficacement réfuté naguère par Tzvetan Todorov, et il prive de pouvoir non seulement le narrateur mais encore le lecteur. Plutôt, si je peux me permettre une

19 Voir Kaja SILVERMAN, The Acoustic Mirror: The Female Voice in Psychoanalysis and Cinema. Bloomington, IN : Indiana University Press 1988. 
petite recommandation, ce serait une attitude post-structuraliste à l'intérieur de la narratologie qui reste ancrée dans la pensée structurale. Une attitude, en d'autres mots, qui ne saute pas au-delà de ce dont on est loin d'avoir exploité toutes les possibilités, sans pour autant absorber les conséquences dans leur entier. Pour parler dans le champ métaphorique d'un autre grand producteur de narration, François Rabelais, je souhaiterais aux narratologues appliquant un peu d'éclectisme contrôlé ; en d'autres mots, de manger, de manger bien : régalez-vous, mais mâchez bien, pour que chaque morceau soit bien digéré, pour qu'aucun ne reste bloqué sur place. D'où l'importance de la faute de grammaire dans mon titre. 